\title{
Oculopharyngeal muscular dystrophy
}

INSERM

\section{Source}

INSERM. (1999). Orphanet: an online rare disease and orphan drug data base.

Oculopharyngeal muscular dystrophy. ORPHA:270

Oculopharyngeal muscular dystrophy (OPMD) is an adult-onset prog ressive myopathy characterized by progressive eyelid ptosis, dysphagia, dysarthria and proximal limb weakness. 